\title{
A Lagrangian Half-Quadratic Approach to Robust Estimation and its Applications to Road Scene Analysis
}

\author{
Jean-Philippe Tarel $^{\mathrm{a}}$, Pierre Charbonnier ${ }^{\mathrm{b}}$ \\ ${ }^{a} L C P C, 58$ Bd Lefebvre, 75015 Paris, France \\ ${ }^{b}$ ERA 27 LCPC, 11 rue Jean Mentelin, B.P. 9, 67035, Strasbourg, France
}

\begin{abstract}
We consider the problem of fitting linearly parameterized models, that arises in many computer vision problems such as road scene analysis. Data extracted from images usually contain non-Gaussian noise and outliers, which makes non-robust estimation methods ineffective. In this paper, we propose an overview of a Lagrangian formulation of the Half-Quadratic approach by, first, revisiting the derivation of the well-known Iterative Re-weighted Least Squares (IRLS) robust estimation algorithm. Then, it is shown that this formulation helps derive the so-called Modified Residuals Least Squares (MRLS) algorithm. In this framework, moreover, standard theoretical results from constrained optimization can be invoked to derive convergence proofs easier. The interest of using the Lagrangian framework is also illustrated by the extension to the problem of the robust estimation of sets of linearly parameterized curves, and to the problem of robust fitting of linearly parameterized regions. To demonstrate the relevance of the proposed algorithms, applications to lane markings tracking, road sign detection and recognition, road shape fitting and road surface 3D reconstruction are presented.
\end{abstract} Key words: Non Convex problem, Constrained Optimization, Robust 
Estimators, Image Analysis, Stereovision, Linear generative model.

\section{Introduction}

As in many scientific activities, a very common approach to image analysis 3 involves collecting $n$ observations $\left(x_{1}, y_{1}\right), \cdots,\left(x_{n}, y_{n}\right)$ that take their values ${ }_{4}$ in $\mathbb{R}^{p} \times \mathbb{R}$, and then finding the model that best fits these data. The simplest 5 regression model is the linear one:

$$
y_{i}=X\left(x_{i}\right)^{t} A+b_{i} \quad i=1, \ldots n
$$

6 where $A=\left(a_{l}\right)_{0 \leq l \leq d}$ is the vector of (unknown) model parameters, $X\left(x_{i}\right)=$

$7\left(f_{l}\left(x_{i}\right)\right)_{0 \leq l \leq d}$ collects the values of some basis of real functions at locations $8 x_{i}$ and $b_{i}$ is the random measurement noise. $X\left(x_{i}\right)$ is also called design of 9 the measurement (or experiment) [1]. We assume that the residuals, $b_{i}$, are applications, residuals are most of the time non-Gaussian and thus some gross errors, called outliers may be observed.

Outliers pose a threat on parametric regression under the linear generative model (1) in the sense of ordinary Gaussian Maximum-Likelihood estimation. To alleviate their biasing effect on estimation, Huber proposed in [2] to use non-Gaussian Maximum-Likelihood type estimators, a.k.a. Mestimators. Bounding the resulting non-quadratic energy with parabolas leads to the so-called Iterative Re-weighted Least Squares (IRLS or IRWLS) or to Modified Residuals Least Squares (MRLS), depending on the analytic form of the parabola. Unfortunately, although the theory of robust estimation was already known in statistics as early as the mid 80s, it did not cross the barrier of communities. 
In image reconstruction, the work of the Geman brothers [3-5] for edgepreserving regularization was integrated and extended in $[6,7]$ under the name of Half-Quadratic regularization. The direct connection between Mestimation and Half-Quadratic theory was pointed out in [8]. More recently, Lange [9] proposed an interpretation of these algorithms as optimization transfer or bounded optimization algorithms, a particular class of ConvexConcave procedures [10] to which the Expectation Maximization (EM) algorithm also belongs. An interpretation of Half-Quadratic algorithms as EM algorithms was also proposed in [11].

In this paper, we revisit a derivation of Half-Quadratic algorithms which is based on a Lagrangian formalism. The Lagrangian Half-Quadratic approach was developed in a series of papers which are here summarized for a unified presentation. Our goal in this article is to show that the Lagrangian Half-quadratic approach offers two non-negligible advantages over existing derivations. First, it eases convergence proofs since we can invoke standard results from the theory of constrained optimization. This is illustrated by the derivation of the classical IRLS algorithm, as presented first in [16] and by the derivation of the less known MRLS algorithm, as first explain here. Second, it helps derive new algorithms, for e.g. simultaneous regression of multiple curves (first presented in [23] and detailed in $[12,13]$ ) or for linearly parameterized region fitting (first presented in [33] for road surface segmentation). Moreover, we show that the MRLS and IRLS algorithms can be used with advantages in many computer vision problems as illustrated with two non-obvious and original application examples in the context of road scene analysis: detection and recognition of traffic signs (previously 
49 in [30] for the road surface case). difficult problems, such as: logical conditions,

- road pavement detection in images,

\section{Robust Parameter Estimation} (pdf) of the residual, $b$ in the form:

The organization of the paper is as follows: in Section 2, the Lagrangian formulation is presented and is applied to the derivation of the IRLS and MRLS algorithms. We also present a family of potential functions, and a continuation heuristic which helps convergence to a satisfactory local minimum. Various applications, involving extensions of the proposed framework, in the field of road scene analysis are proposed in Section 3. We also address

- multiple marking lanes detection and tracking under adverse meteoro-

- road sign detection and recognition,

- 3D road shape reconstruction using stereovision.

As explained in $[12,13]$, the derivation of the robust estimator can be obtained using Lagrange's formulation which leads to the same algorithms as those obtained by the Half-Quadratic and M-estimation approaches. As in these approaches, we focus on a symmetric probability density functions

$$
p(b) \propto \frac{1}{s} e^{-\frac{1}{2} \phi\left(\left(\frac{b}{s}\right)^{2}\right)}
$$


where $\propto$ denotes the equality up to a factor, and $s$ is the scale of the noise.

With the Maximum-Likelihood Estimation (MLE) method, the problem of estimating the linear model (1) under noise (2) is set as the minimization w.r.t. $A$ of the error:

$$
e_{R}(A)=\frac{1}{2} \sum_{i=1}^{i=n} \phi\left(\left(\frac{y_{i}-X_{i}^{t} A}{s}\right)^{2}\right) .
$$

To solve this problem, as in the Half-Quadratic approach $[4,7], \phi(t)$ should fulfill the following hypotheses:

- H0: $\phi$ is defined and continuous on $[0,+\infty[$ as well as its first and second derivatives,

- H1: $\phi^{\prime}(t)>0$ (thus $\phi$ is increasing),

- $\mathbf{H 2 :} \phi^{\prime \prime}(t)<0$ (thus $\phi$ is concave).

Specifically for the derivation of the MRLS algorithm, a fourth hypothesis on $\phi(t)$ is required:

- H3: $\phi^{\prime}(t) \leq 1$ ( $\phi^{\prime}$ is bounded $)$.

As stated in [2], the role of $\phi$ is to saturate the error in case of a large scaled residual $\left|b_{i}\right|=\left|y_{i}-X_{i}^{t} A\right|$, and thus to lower the importance of outliers.

The scale parameter, $s$, sets the residual value from which noisy data points have a good chance of being considered as outliers.

The Lagrangian formulation consists in first rewriting the problem as a constrained optimization problem that is solved by the search for a saddle point of the associated Lagrange function. Then, the algorithm is obtained by alternated minimizations of the dual function. As we will see, for (3), 
two algorithms can be derived: the first derived algorithm corresponds to the well-known algorithm named Iterative Re-weighted Least Squares (IRLS) see Section 2.1, and the second known as the somewhat less popular Modified Residuals Least Squares (MRLS) see Section 2.2.

\subsection{Iterative Re-weighted Least Square (IRLS)}

First, we rewrite the minimization of $e_{R}(A)$ as the maximization of $-e_{R}$. This will allow us later to write $-e_{R}(A)$ as the extremum of a convex function rather than a concave one, since the negative of a concave function is convex. Second, we introduce the auxiliary variables $w_{i}=\left(\frac{y_{i}-X_{i}^{t} A}{s}\right)^{2}$. These variables are needed to rewrite $-e_{R}(A)$ as the value achieved at the minimum of a constrained problem. This apparent complication is in fact valuable since it allows us to introduce a Lagrange function. Indeed using $\mathbf{H 1},-e_{R}(A)$ can be seen as the value achieved by the minimization w.r.t. $W=\left(w_{i}\right)_{1 \leq i \leq n}$ of:

$$
E_{I R L S}(A, W)=\frac{1}{2} \sum_{i=1}^{i=n}-\phi\left(w_{i}\right),
$$

subject to $n$ constraints $w_{i}-\left(\frac{y_{i}-X_{i}^{t} A}{s}\right)^{2} \leq 0$, for any $A$. This sub-problem is well-posed because it is a minimization of a convex function subject to linear constraints. Therefore using Kuhn and Tucker's classical theorem [14], if a solution exists, the minimization of $E_{I R L S}(A, W)$ w.r.t. $W$ is equivalent to search from the unique saddle point of the Lagrangian:

$$
L_{I R L S}\left(A, W, \lambda_{i}\right)=\frac{1}{2} \sum_{i=1}^{i=n}-\phi\left(w_{i}\right)+\lambda_{i}\left(w_{i}-\left(\frac{y_{i}-X_{i}^{t} A}{s}\right)^{2}\right),
$$

where $\lambda_{i}$ are Kuhn-Tucker multipliers $\left(\lambda_{i} \geq 0\right)$. 
The derivatives of the Lagrange function $L_{I R L S}\left(A, W, \lambda_{i}\right)$ w.r.t. the auxiliary variables $W$, the unknown variables $A$, and the Khun-Tucker multipliers $\lambda_{i}$ are set to zero to obtain the IRLS algorithm:

1. Initialize $A^{0}$, and set $k=1$,

2. For all $1 \leq i \leq n$, compute the auxiliary variables $w_{i}^{k}=\left(\frac{y_{i}-X_{i}^{t} A^{k-1}}{s}\right)^{2}$ and the weights $\lambda_{i}^{k}=\phi^{\prime}\left(w_{i}^{k}\right)$,

3. Solve the linear system $\sum_{i=1}^{i=n} \lambda_{i}^{k} X_{i} X_{i}^{t} A^{k}=\sum_{i=1}^{i=n} \lambda_{i}^{k} X_{i} y_{i}$,

4. If $\left\|A^{k}-A^{k-1}\right\|>\epsilon$ then increment $k$ and go to 2 , else $A_{I R L S}=A^{k}$.

As detailed in [13], this algorithm can be derived rigorously from the alternated minimization of the dual error associated to the Lagrange function $L_{I R L S}\left(A, W, \lambda_{i}\right)$. Moreover, it can be shown that such an algorithm always strictly decreases the dual function if the current point is not a stationary point (i.e. a point where the first derivatives are all zero) of the dual function $[15]$.

\subsection{Modified Residuals Least Squares}

The IRLS algorithm is into a multiplicative form, but there also exists an algorithm based on an additive Half-Quadratic development called Modified Residuals Least Squares (MRLS), which can be convenient for particular applications as illustrated in Section 3.2. The Lagrangian formulation can also be used to derive the MRLS algorithm and to prove its convergence towards a local minimum. Again, we introduce auxiliary variables and rewrite $e_{R}(A)$ as the value achieve as the search for a saddle point of the associated Lagrange's function. Then, the algorithm is obtained by alternated minimization of the dual function. 
Let us introduce function $f(b)=b^{2}-\phi\left(b^{2}\right)$ defined on $[0,+\infty[$, and thus rewrite $e_{R}(A)$ as:

$$
e_{R}(A)=\frac{1}{2} \sum_{i=1}^{i=n}\left(\frac{y_{i}-X_{i}^{t} A}{s}\right)^{2}-f\left(\left|\frac{y_{i}-X_{i}^{t} A}{s}\right|\right) .
$$

This will allow us to later write $e_{R}(A)$ as the value achieved at the minimum of a convex function for any $A$. First, we have $f^{\prime}(b)=2 b\left(1-\phi^{\prime}\left(b^{2}\right)\right)$ and thus $f$ is increasing as required by H3. Moreover, we have $f^{\prime \prime}(b)=$ $2\left(1-\phi^{\prime}\left(b^{2}\right)-2 \phi^{\prime \prime}\left(b^{2}\right) b^{2}\right)$, and thus $f^{\prime \prime}(b) \geq 0$ using $\mathbf{H 2}$ and H3. Second, we introduce the auxiliary variables $\omega_{i}=\left|\frac{y_{i}-X_{i}^{t} A}{s}\right|$. These variables are needed to rewrite $e_{R}(A)$ as the value achieved at the minimum of a constrained problem. Indeed, using the fact that $f$ is increasing, the second term $\sum_{i=1}^{i=n} f\left(\left|\frac{y_{i}-X_{i}^{t} A}{s}\right|\right)$ of $e_{R}(A)$ can be seen as the value achieved at the minimization with respect to $\Omega=\left(\omega_{i}\right)_{1 \leq i \leq n}$ of $E_{M R L S}(A, \Omega)=\sum_{i=1}^{i=n} f\left(\omega_{i}\right)$, subject to $n$ constraints $\left|\frac{y_{i}-X_{i}^{t} A}{s}\right|-\omega_{i} \leq 0$. This last sub-problem is well-posed because it is a minimization of a convex function subject to linear constraints w.r.t. $\Omega$. Therefore using the classical Kuhn and Tucker's theorem [14], if a solution exists, the minimization of $E_{M R L S}(A, \Omega)$ with respect to $\Omega$ is equivalent to the search of the unique saddle point of the Lagrangian of the sub-problem:

$$
L_{M R L S}\left(A, \Omega, \lambda_{i}\right)=\sum_{i=1}^{i=n} f\left(\omega_{i}\right)+\lambda_{i}\left(\left|\frac{y_{i}-X_{i}^{t} A}{s}\right|-\omega_{i}\right)
$$

More formally, we have proved for any $A$ :

$$
\sum_{i=1}^{i=n} f\left(\left|\frac{y_{i}-X_{i}^{t} A}{s}\right|\right)=\min _{\omega_{i}} \max _{\lambda_{i}} L_{M R L S}\left(A, \Omega, \lambda_{i}\right) .
$$

Using the saddle point property, we can change the order of variables $\omega_{i}$ and $\lambda_{i}$ in (4). $L_{M R L S}\left(A, \Omega, \lambda_{i}\right)$ being convex with respect to $\Omega$, it is equivalent 
to search for a minimum of $L_{M R L S}\left(A, \Omega, \lambda_{i}\right)$ with respect to $\Omega$ and to have its first derivatives zero. Thus, we deduce $\lambda_{i}=f^{\prime}\left(\omega_{i}\right)$. Recalling that $f^{\prime}$ is increasing, this last equation can be used to substitute $\omega_{i}$ in $L$ :

$$
\sum_{i=1}^{i=n} f\left(\left|\frac{y_{i}-X_{i}^{t} A}{s}\right|\right)=\max _{\lambda_{i}} L_{M R L S}\left(A, f^{\prime-1}\left(\lambda_{i}\right), \lambda_{i}\right)
$$

Therefore, we deduce that the original problem is equivalent to the following minimization:

$$
\min _{A} e_{R}(A)=\frac{1}{2} \min _{A, \lambda_{i}} \sum_{i=1}^{i=n}\left(\frac{y_{i}-X_{i}^{t} A}{s}\right)^{2}-L_{M R L S}\left(A, f^{\prime-1}\left(\lambda_{i}\right), \lambda_{i}\right)
$$

The function $\mathcal{E}\left(A, \lambda_{i}\right)=\frac{1}{2} \sum_{i=1}^{i=n}\left(\frac{y_{i}-X_{i}^{t} A}{s}\right)^{2}-\frac{1}{2} L_{M R L S}\left(A, f^{\prime-1}\left(\lambda_{i}\right), \lambda_{i}\right)$ is the dual error of the original problem. Notice that the dual error $\mathcal{E}$ is rather simple with respect to $A$, contrary to the original error $e_{R}$. Indeed, the dual error can be rewritten as:

$$
\mathcal{E}\left(A, \lambda_{i}\right)=\frac{1}{2} \sum_{i=1}^{i=n}\left(\left|\frac{y_{i}-X_{i}^{t} A}{s}\right|-\frac{\lambda_{i}}{2}\right)^{2}+\xi\left(\lambda_{i}\right)
$$

with $\xi\left(\lambda_{i}\right)=-\frac{\lambda_{i}^{2}}{4}-f\left(f^{\prime-1}\left(\lambda_{i}\right)\right)+\lambda_{i} f^{\prime-1}\left(\lambda_{i}\right)$

Taking its second derivatives, we deduce that $\mathcal{E}$ is convex with respect to A. $\mathcal{E}$ is also convex with respect to $\lambda_{i}$ since $f$ is convex and $\frac{\partial^{2} \mathcal{E}}{\partial \lambda_{i}^{2}}=\frac{1}{f^{\prime \prime}\left(f^{\prime-1}\left(\lambda_{i}\right)\right)} \geq$ 0 . However, when $e_{R}\left(b^{2}\right)$ is not convex, $\mathcal{E}$ may not be convex with respect to both $A$ and $\lambda_{i}$. In such a case, $\mathcal{E}\left(A, \lambda_{i}\right)$ does not have a unique minimum.

An alternate minimization of the dual error $\mathcal{E}$, with respect to $A$ and each $\lambda_{i}$, involves the sign of $y_{i}-X_{i}^{t} A$, and leads to the modified residual iterative algorithm, already derived in the Half-Quadratic and M-estimators approaches: 
1. Initialize $A^{0}$, and set $k=1$,

2. For all $1 \leq i \leq n$, compute the auxiliary variables $\omega_{i}^{s i g n, k}=\frac{y_{i}-X_{i}^{t} A^{k-1}}{s}$ and the weights $\lambda_{i}^{\text {sign }, k}=\omega_{i}^{\text {sign }, k}\left(1-\phi^{\prime}\left(\omega_{i}^{\text {sign, } k^{2}}\right)\right)$,

3. Solve the linear system $\sum_{i=1}^{i=n} X_{i} X_{i}^{t} A^{k}=\sum_{i=1}^{i=n} X_{i}\left(y_{i}-s \lambda_{i}^{s i g n, k}\right)$,

4. If $\left\|A^{k}-A^{k-1}\right\|>\epsilon$ then increment $k$ and go to 2 , else $A_{M R L S}=A^{k}$.

In this algorithm we introduce the two notations $\omega_{i}^{\text {sign, } k}=\omega_{i}^{k} \operatorname{sign}\left(y_{i}-\right.$ $\left.X_{i}^{t} A^{k-1}\right)$ and $\lambda_{i}^{s i g n, k}=\lambda_{i}^{k} \operatorname{sign}\left(y_{i}-X_{i}^{t} A^{k-1}\right)$, which are signed version of the auxiliary variables $\omega_{i}$ and $\lambda_{i}$. Like in the previous section, it can be shown that this alternate minimization of $\mathcal{E}$ always strictly decreases the dual function, if the current point is not already a stationary point [15]. Following the lines of [12], it can then be deduced that the proposed algorithm is globally convergent, i.e., it converges towards a local minimum of $e_{R}(A)$ for all initial $A_{0}$ 's which are not a maximum or a saddle point of $e_{R}(A)$.

\subsection{Non-Gaussian Noise Model}

We are interested in a parametric family of functions for noise modeling, in the form of (2) that allows a continuous transition between different kinds of useful probability distributions. We thus focus on a simple parametric probability density functions (pdf) of the form:

$$
S_{\alpha, s}(b) \propto \frac{1}{s} e^{-\frac{1}{2} \phi_{S_{\alpha}}\left(\left(\frac{b}{s}\right)^{2}\right)}
$$

where the associated $\phi$ function is $\phi_{S_{\alpha}}(t)=\frac{1}{\alpha}\left((1+t)^{\alpha}-1\right)$. This is the so-called Smooth Exponential Family (SEF) introduced in $[12,16,17]$ which is suitable for the IRLS and MRLS algorithms, since (for $\alpha<1$ ) it satisfies the four hypotheses $\mathbf{H 0}, \mathbf{H 1}, \mathbf{H 2}$ and $\mathbf{H 3}$. 


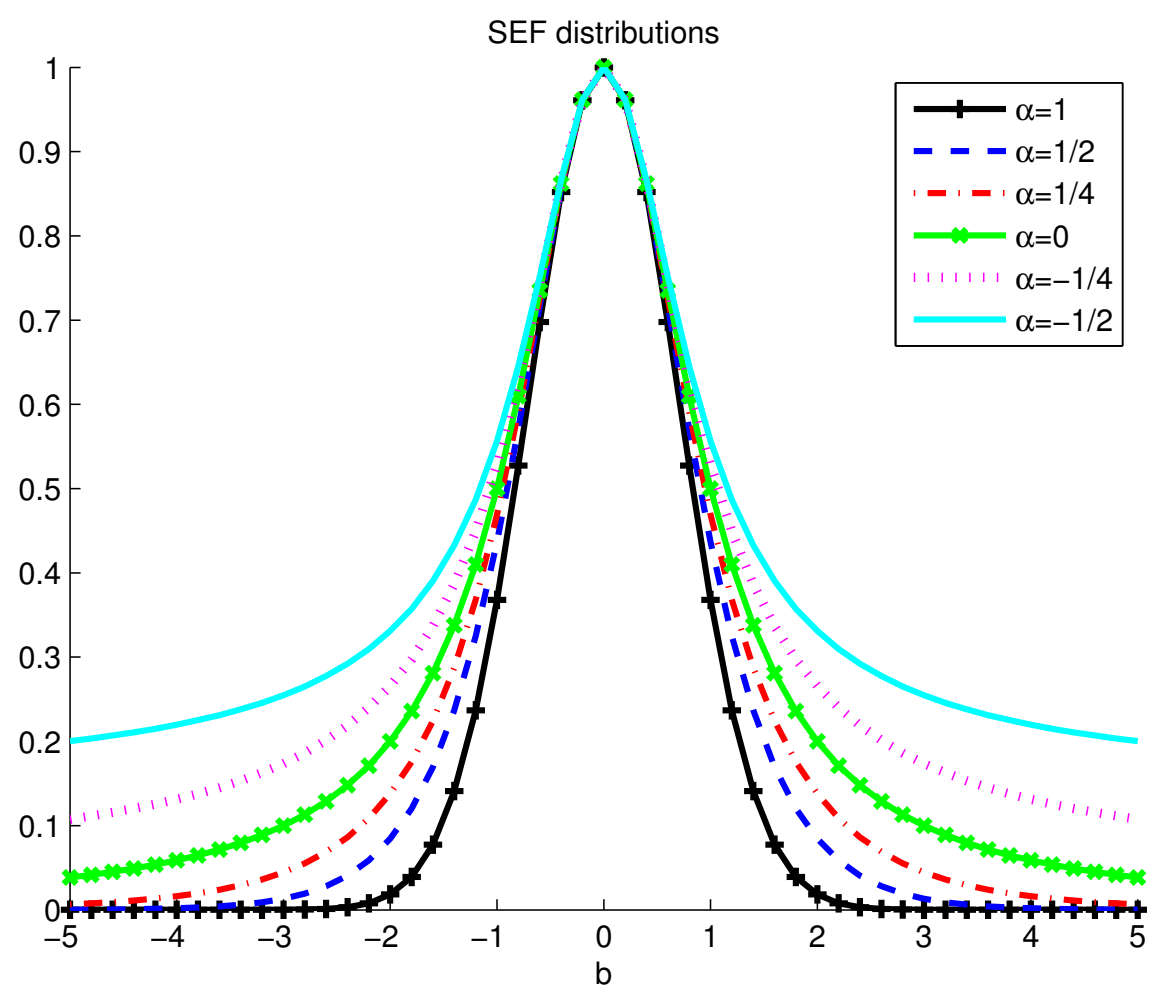

Figure 1: SEF noise models, $S_{\alpha, s}$ for $s=1$ and different values of $\alpha$. Notice how tails are getting heavier as $\alpha$ decreases.

The parameter $\alpha$ allows a continuous transition between well-known statistical laws such as Gauss $(\alpha=1)$, smooth Laplace $\left(\alpha=\frac{1}{2}\right)$ and Geman \& McClure [18] $(\alpha=-1)$. These laws are shown on Figure 1. Note that, for $\alpha<0, S_{\alpha, s}$ can be always normalized on a bounded support, so it can still be considered as a pdf. In the smooth exponential family, when $\alpha$ is decreasing, the probability to observe large, not to say very large errors (outliers), increases.

In the IRLS and MRLS algorithms, the weights $\lambda_{i}$ are a function of $\phi^{\prime}(t)$. 
For the SEF, this function is simply $\phi_{S_{\alpha}}^{\prime}(t)=(1+t)^{\alpha-1}$. Notice that while the pdf is not defined when $\alpha=0$, the corresponding $\phi^{\prime}(t)$ does and that it corresponds in fact to the Cauchy distribution or Student's t-distribution [17].

It can be shown, see $[12,17]$, that the breakdown point of SEF estimators is increasing towards the maximum achievable value, that is $50 \%$, as $\alpha \in$ ]0,0.5] decreases. The maximum goes to $50 \%$ when $\alpha \rightarrow 0$.

\subsection{Graduated Non-Convexity (GNC)}

The function $\phi^{\prime}(t)$, used in the IRLS and MRLS algorithms, becomes more sharply peaked and heavily tailed as $\alpha$ decreases. As a consequence, the lower the $\alpha$, the lower the effect of outliers on the result. Therefore, the algorithm produces a more robust fitting. However, when $\alpha$ decreases, the error function $e_{R}(A)$ becomes less and less smooth. If $\alpha=1$, the cost function is a paraboloid and thus there exists a unique global minimum. For $\alpha$ between 1 and 0.5 , the cost function is convex w.r.t. $A$ and thus there still exists a unique global minimum. By decreasing $\alpha$ to values lower than $\frac{1}{2}$, local minima appear. This is illustrated in Figure 2 where the error function $e_{R}(A)$ is shown for four decreasing values of $\alpha$.

Following the principle of the GNC method [19], the localization property of the robust fit w.r.t. the decreasing parameter $\alpha$ can be used to converge toward a local minimum close to the global one. Convexity is first enforced using $\alpha=1$ or $\alpha=0.5$. Then, a sequence of fits with a stepwise decreasing $\alpha$ is performed in continuation, i.e. each time using the current output fit as an initial value for the next fitting step. Of course, as detailed in [20], $\alpha$ must be decreased slowly, otherwise the curve fitting algorithm might be trapped into a shallow local minimum far from the global one. 


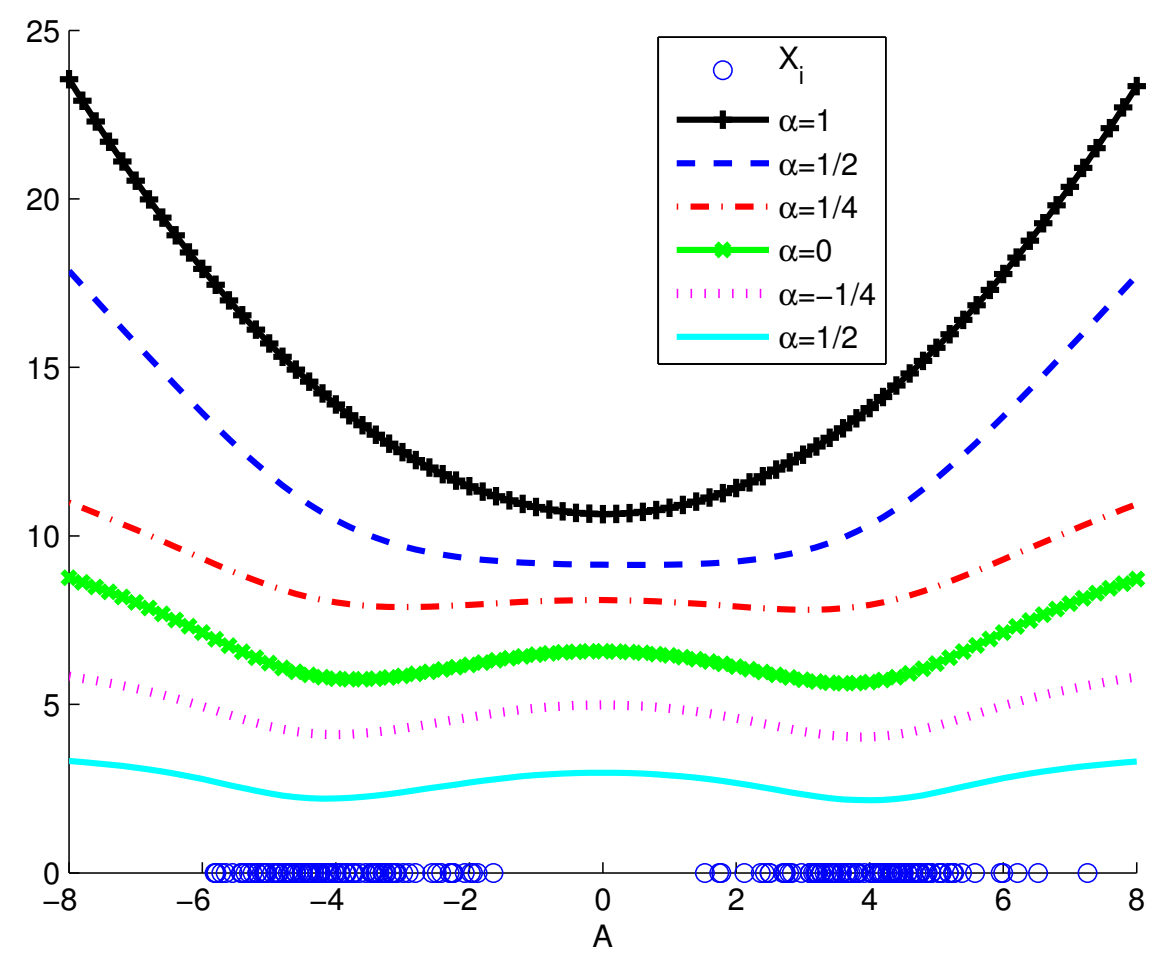

Figure 2: The error function $e_{R}(A)$ for an example of scalar data with two clusters, for different values of $\alpha$. Notice the progressive appearance of the second minimum while $\alpha$ decreases.

\section{Applications and extensions in road scene analysis}

3.1. Lane-markings Fitting

In our approach of lane marking detection, road marking elements are

first extracted from a road scene image using one of the algorithm compared in [21]. Then, the centers of marking elements are fitted with a curve using an IRLS algorithm. As explained in [22], it is possible to use different curve families to model the shape of a lane marking, such as polynomial curves 

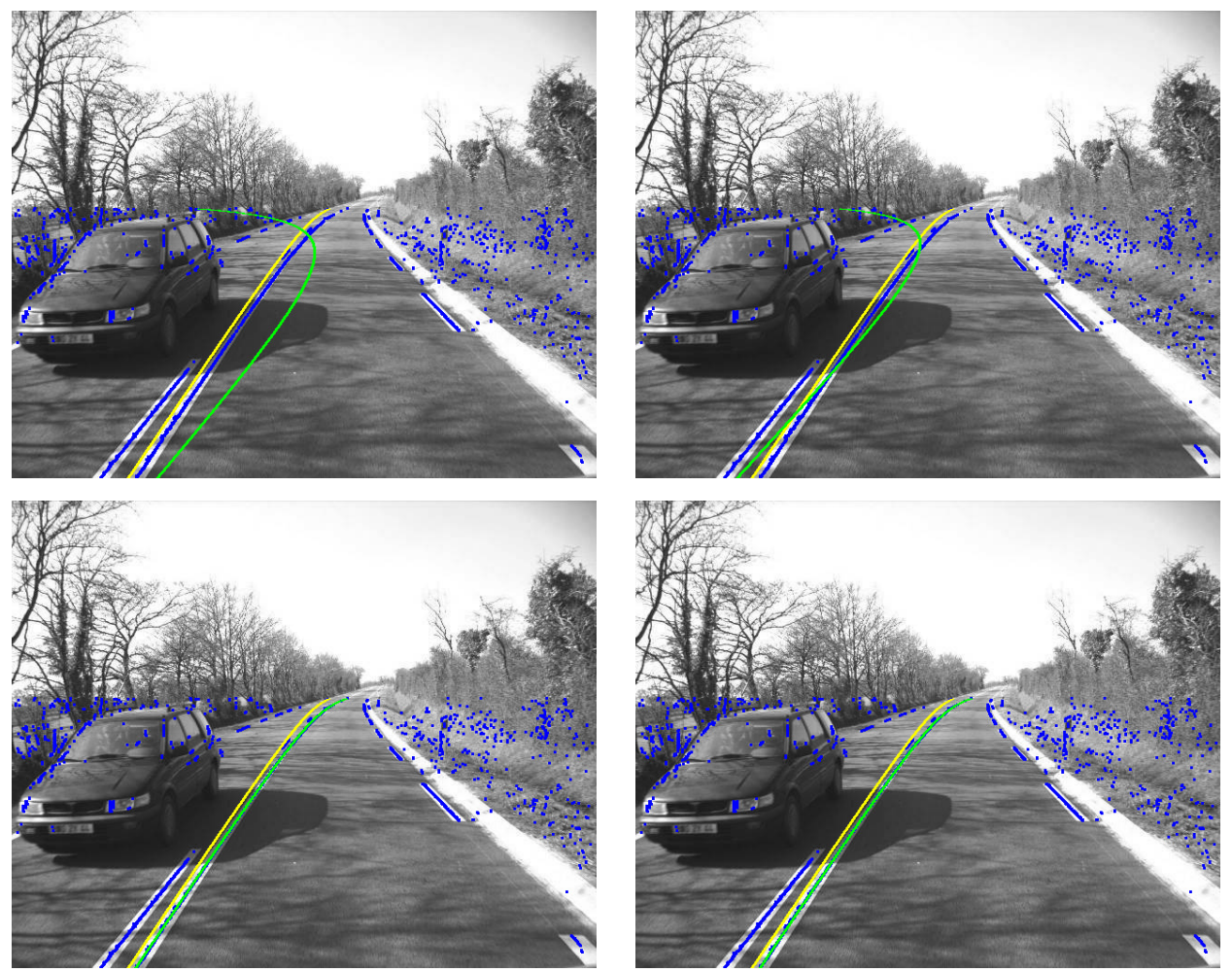

Figure 3: Fitting on a real image assuming (from left to right, up to down) Gauss, smooth Laplace, Cauchy, and Geman $\&$ McClure [18] noise pdfs. Data points are shown in blue, yellow lines are the initial $A^{0}$ and green lines are the fitting results.

or hyperbolic polynomial curves, which better fit road edges on long range distances.

Figure 3 illustrates the importance of robust fitting in images with many outliers. The yellow line depicts the initial $A^{0}$ obtained from the previous image. The green ones are the fitting results $A_{I R L S}$, assuming respectively Gauss, smooth Laplace, Cauchy, and Geman \& McClure [18] noises (as defined in Section 2.3). A correct fit is achieved only with the last two pdf's, which correspond to non-convex errors $e_{R}(A)$. The scale $s$ is fixed to 4 pixels 
from the analysis of the residuals on a ground-truth database.
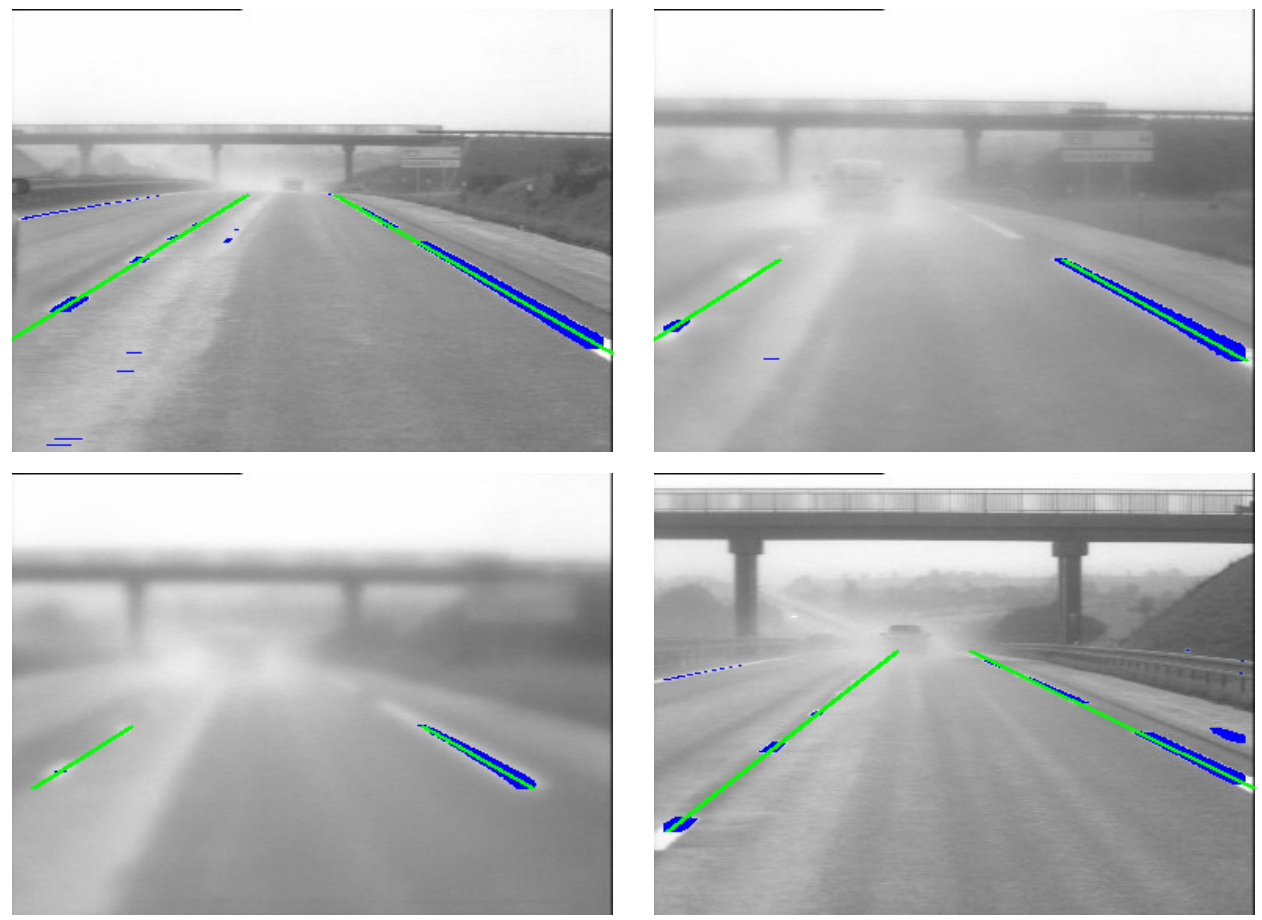

Figure 4: Fitting of two lines along a sequence with rain and visibility difficulties.

As visible on Figure 3, several lane markings can be seen in a road image.

We formulate the problem of robustly estimating in a simultaneous fashion $m$ linearly parameterized curves $A_{j}$, whose parameters may be concatenated in a single vector $A=\left(A_{j}\right)_{j=1 \ldots m}$, as the optimization of:

$$
e_{M R}(A)=-\sum_{i=1}^{i=n} \log \sum_{j=1}^{j=m} e^{-\frac{1}{2} \phi\left(\left(\frac{y_{i}-X_{i}^{t} A_{j}}{s}\right)^{2}\right)} .
$$

The Lagrangian formalism eases deriving from the minimization of (7) the so-called Simultaneous Robust Multiple Fitting (SRMF) algorithm [12]:

1. Choose an initial estimate $A^{0}$, and initialize loop index to $k=1$. 
2. For each $1 \leq j \leq m$, compute vector $W_{j}^{k}=\frac{1}{s}\left(X^{t} A_{j}^{k-1}-Y\right)$ and matrix $B_{j}^{k}=\operatorname{diag}\left\{\frac{e^{-\phi\left(w_{j i}^{k}\right)}}{\sum_{j=1}^{j=m} e^{-\phi\left(w_{j i}^{k}\right)}} \phi^{\prime}\left(w_{j i}^{k}\right)\right\}_{i=1 \ldots n}$.

3. For each $1 \leq j \leq m$, solve $X B_{j}^{k} X^{t} A_{j}^{k}=X B_{j}^{k} Y$.

4. If $\left\|A^{k}-A^{k-1}\right\|>\epsilon$ then increment $k$ and go to 2 , else $A_{S R M F}=A^{k}$.

With notations $X=\left(X_{i}\right)_{1 \leq i \leq n}, Y=\left(y_{i}\right)_{1 \leq i \leq n}$ and $W_{j}^{k}=\left(w_{j i}^{k}\right)_{1 \leq i \leq n}$. More details on the derivation and on the use of the SRMF algorithm may be found in [12], where the convergence to a local minimum is proved. Notice that the IRLS algorithm may be seen as a special case of the SRMF algorithm with $m=1$. Moreover, note that the SRMF weights incorporate a probability ratio, which is customary used in classification algorithms as a membership function.

The tracking of several curves along a sequence of images is performed by embedding the SRMF algorithm within a Kalman filter (see [13, 16, 23] for details) as illustrated in Figure 4.

\subsection{Appearance-based road sign detection and recognition}

Appearance-based models have met unquestionable success in the field of object detection and recognition, since the early 1990s [24]. In such models, objects are represented by their raw brightness or color vector, without any feature extraction. To capture non-linear appearance variations while using a linear generative model, we proposed in $[25,26]$ an approach based on probabilistic principal component analysis [27]. More specifically, any observed image $y$ may be reconstructed on a relatively low-dimensional basis of functions, that span the eigenspace of the covariance matrix obtained from a set of representative images: $y=\mu+X^{t} A+b$, where $\mu$ is the sample mean 
of the learning set. This is a special case of model (1) where the $X_{i}$ 's are not analytical but statistical basis functions. Figure 5 shows an example of sample images and the corresponding basis vectors. Detection and recognition tasks involve estimating the latent variable, $A$. Such a regression task may be performed robustly in the Half-Quadratic framework. In this particular case, the scale variable, $s$, is fixed accordingly to a statistical study of the residuals obtained by reconstructing the (outlier-free) learning image samples [25]. As shown in Figure 5, robust estimation provides both a correct reconstructed image and outliers map. Note that, in this particular case where $X$ is orthogonal and the dimension of the eigenspace is typically about 20, the MRLS algorithm is recommended since it can be more computationally efficient than the IRLS. We shown in [25] that enforcing a well-suited prior to the latent variable is beneficial to the quality of detection. Moreover, we proposed in [26] an original algorithm which handles both robust likelihoods and arbitrary non-parametric priors on $A$ in the framework of Mean Shift [28] (which, besides, is also a Half-Quadratic algorithm [29]). This approach shows very good performance in recognition task on real images.

\subsection{Road profile $3 D$ reconstruction}

Another application of our Lagrangian formalism is road profile reconstruction from stereovision, assuming a rectified geometry. In practice, the left and right views mostly contain the image of the road. The surface of the road in front of the vehicle defines a mapping between the two images. Assuming a polynomial model for the road profile [30], within the disparity space [31], this mapping between a point $(u, v)$ in the left image and a point 


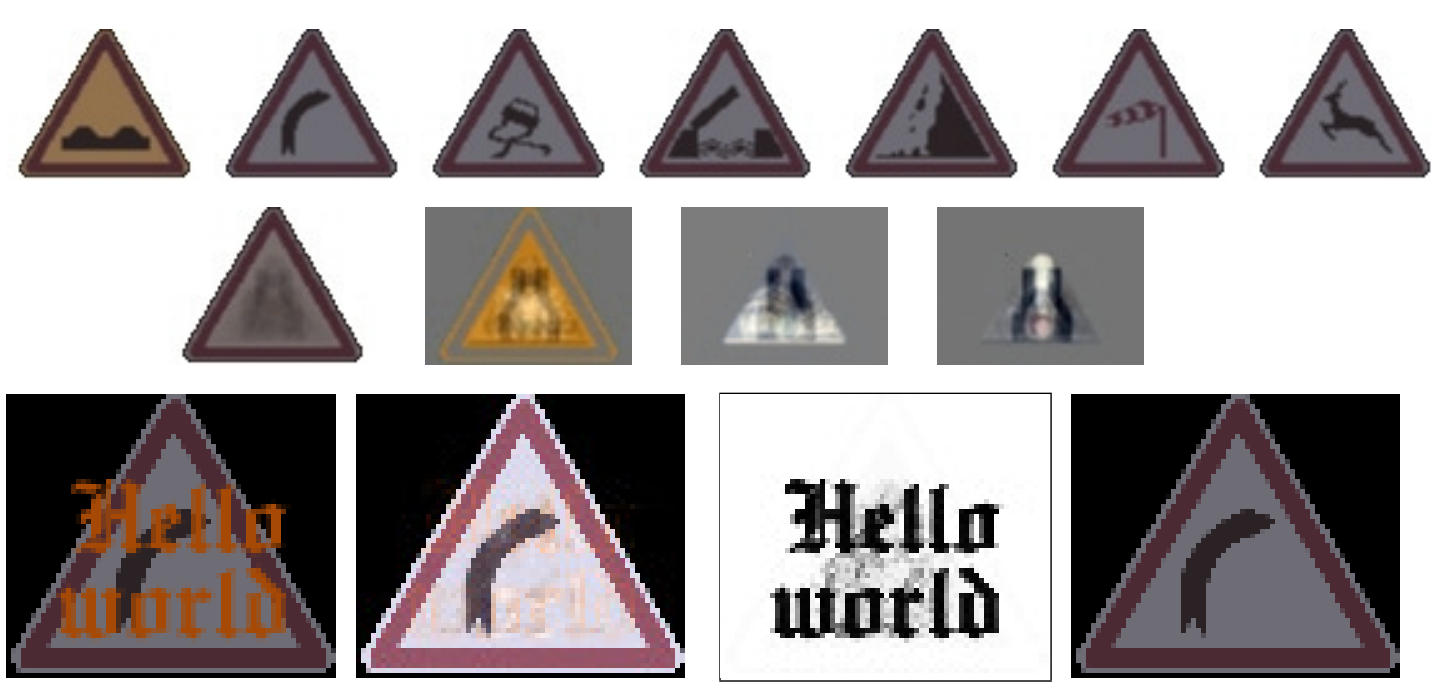

Figure 5: First row: 7 of the $N=43$ images used for learning. Second row: mean image and first 3 eigenvectors obtained by principal component analysis from the learning database. Third row: recognition experiment. From left to right: analyzed image, reconstruction from robustly estimated co-ordinates in eigenspace, outliers map, recognized image.

$\left(u^{\prime}, v^{\prime}\right)$ in the right image is:

$$
\left.\begin{array}{ccc}
u^{\prime}= & u+a_{0}+a_{1} v+\cdots+a_{n} v^{n}=u+X(v)^{t} A \\
v^{\prime}= & v
\end{array}\right\}
$$

where $X(v)=\left(1, v, v^{2}, \cdots, v^{n}\right)^{t}$ is the vector of monomials and $A=\left(a_{0}, \cdots, a_{n}\right)$ is the vector of unknown parameters related to the vertical road profile.

The $3 \mathrm{D}$ reconstruction of the road surface vertical profile needs horizontal correspondences between the edges on the road in the left and right images. Instead of performing first the matching and then the 3D reconstruction, we solve the problem by formulating it as the fitting of linear model $A$ on the set of all possible horizontal correspondences $\left(\left(u, u^{\prime}\right), v\right)$. The robustness 
of the IRLS estimator is thus of major importance, since many of these correspondences are wrong.

More specifically, we formulate in [30] the estimation problem using a Generalized M-estimator as:

$$
e_{R}(A)=\frac{1}{2} \sum_{\left(u, u^{\prime}\right), v} d\left(\left(u, u^{\prime}\right), v\right) \phi\left(\left(\frac{u+X(v)^{t} A-u^{\prime}}{s}\right)^{2}\right),
$$

where $d\left(\left(u, u^{\prime}\right), v\right)$ measures the local similarity in terms of gray or color gradients between the two matched edge pixels $(u, v)$ and $\left(u^{\prime}, v\right)$. The IRLS algorithm resulting from our Lagrangian derivation features an extra factor in the weights, which becomes in that case $\lambda_{\left(u, u^{\prime}\right), v}=d\left(\left(u, u^{\prime}\right), v\right) \phi^{\prime}\left(\left(\frac{u+X(v)^{t} A-u^{\prime}}{s}\right)^{2}\right)$

To improve convergence towards a local minima close to the global one, the optimization is iterated for several scales $s$. At the beginning, $s$ is set to a large value and is then decreased step by step to 1 pixel. This is another kind of GNC based on the use of the scale $s$ rather than on the use of $\alpha$ as described in Section 2.4.

It is important to notice that, in the obtained algorithm, the matching is one-to-several rather than one-to-one. As experimented in [32], one-toseveral correspondence provides better convergence towards an interesting local minimum, and thus outperforms Iterative Closest Point or other oneto-one correspondence algorithms. This improved convergence property is detrimental in terms of computational burden. To avoid penalizing the algorithm, we perform a matching decimation in the spirit of [32]: correspondences such as $u+X(v) A-u^{\prime}$ is larger than $3 s$ are discarded. This decimation is performed only one time at each scale $s$ without a significant lost of accuracy, see [30]. Figure 6 displays the edges of the right image obtained after 

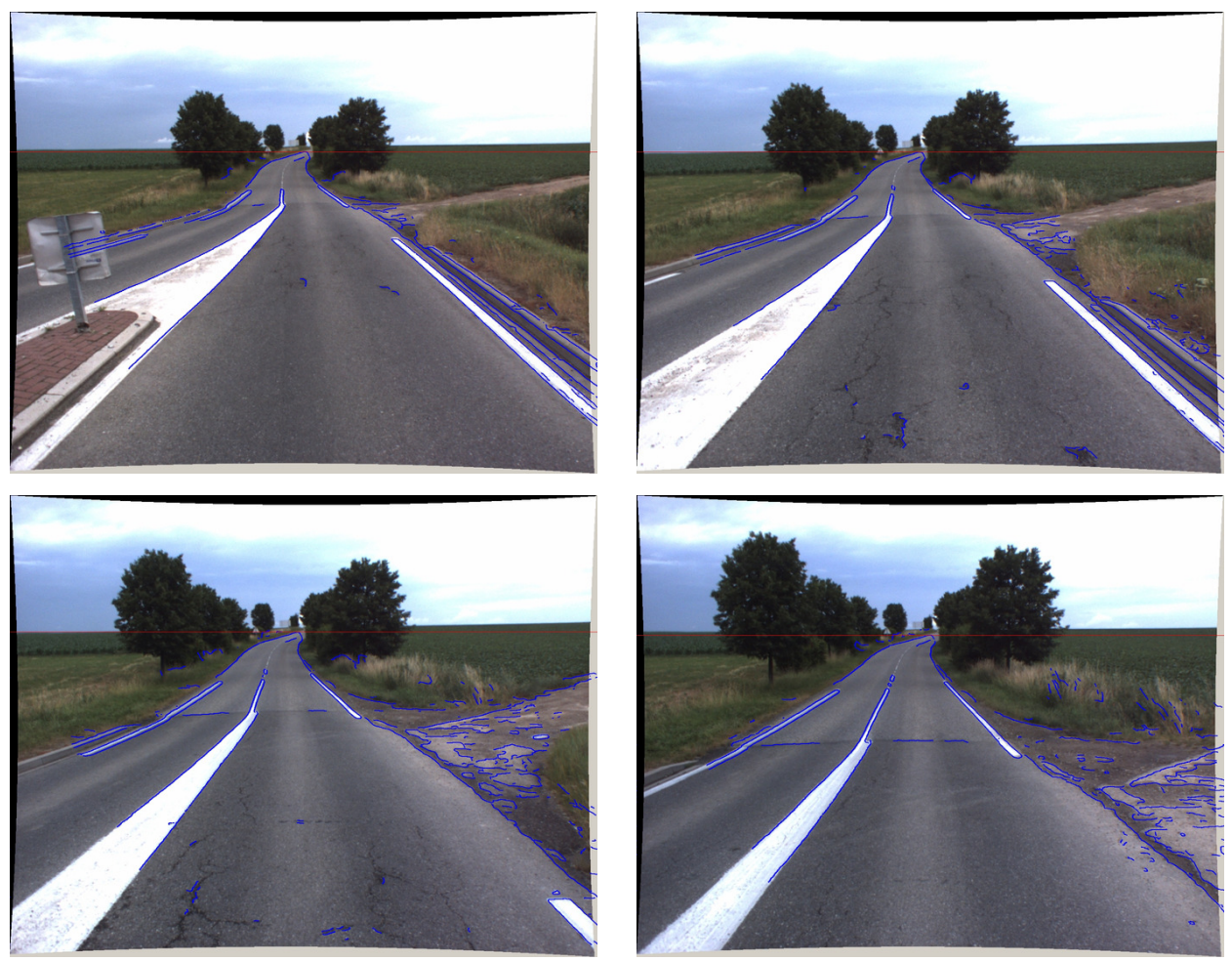

Figure 6: Edges of the right image obtained after alignment on the left image using road profile fitting.

alignment on the left image, showing the quality of the 3D reconstruction of the road vertical profile on four consecutive images.

\subsection{Road region fitting}

Thanks to the Lagrange's formulation, we proposed in [33] a new algorithm for region fitting which is robust to missing data. It assumes linearly parameterized borders and it is applied to the modeling of the road region in a road scene image. As in Section 3.1, we use polynomial or hyperbolic polynomial curves to model the borders of the road region. In vector notations, 

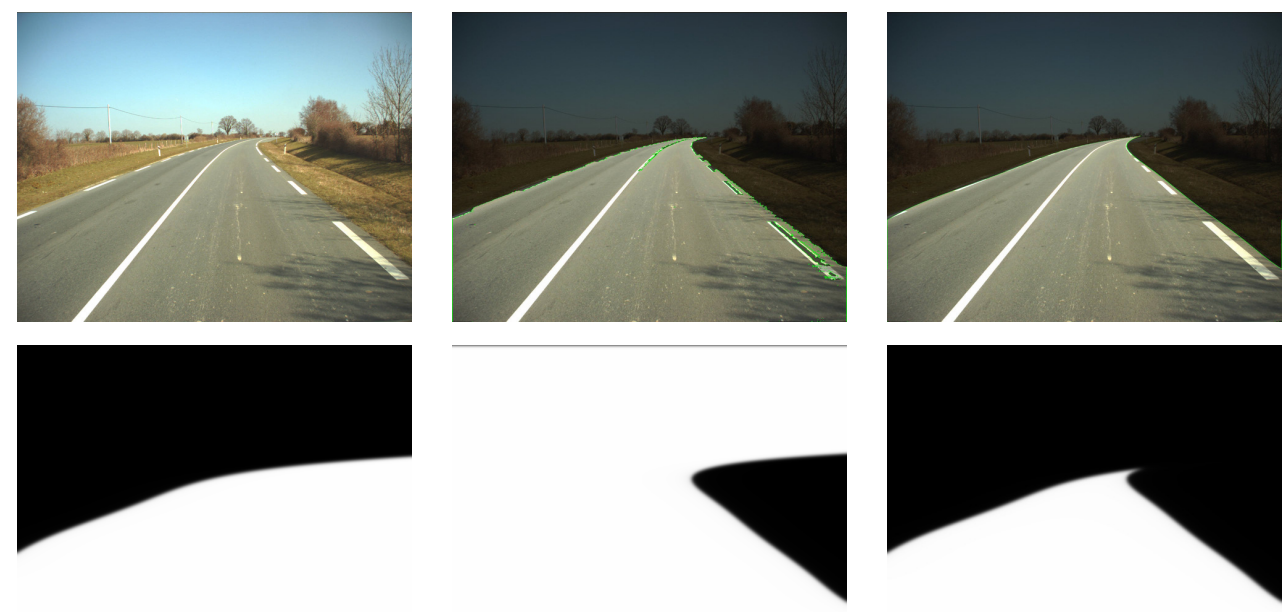

Figure 7: From left to right, first row: original image, color segmentation of the road, resulting region fit on the segmentation, second row: left part of the region, right part of the region, complete resulting region.

right and left borders are thus written as $u_{r}=X(v)^{t} A_{r}$ and $u_{l}=X(v)^{t} A_{l}$.

This region fitting algorithm is obtained as the minimization of the following classical least squares error:

$$
e_{R R F}\left(A_{l}, A_{r}\right)=\iint_{\text {Image }}\left[P(u, v)-\mathcal{R}_{A_{l}, A_{r}}(u, v)\right]^{2} d u d v
$$

between the image $P(u, v)$ which corresponds to the probability of each pixel to belong to the "road" class and the function $\mathcal{R}_{A_{l}, A_{r}}(u, v)$ between 0 and 1 modeling the road region. The probability map is obtained by segmentation of the road colors as detailed in [34], see second image, first row in Figure 7 for an example. This road region is parameterized by $A_{l}$ and $A_{r}$, the parameters of the left and right border curves. Noticing that function $u-X(v)^{t} A$ is defined for all pixel coordinates $(u, v)$, this function is negative on the left of the curve and positive on its right. We thus can use it to define region 
$\mathcal{R}_{A_{l}, A_{r}}$ as:

$$
\mathcal{R}_{A_{l}, A_{r}}(u, v)=\left(g\left(\frac{X(v)^{t} A_{l}-u}{s}\right)+\frac{1}{2}\right)\left(\frac{1}{2}-g\left(\frac{X(v)^{t} A_{r}-u}{s}\right)\right)
$$

where $g$ is an increasing odd function with $g(+\infty)=\frac{1}{2}$. The $s$ parameter tunes the strength of smoothing on the road region borders. The second row in Figure 7 display an example of the first factor in (11), of the second term, and of the result $\mathcal{R}_{A_{l}, A_{r}}$.

By substitution of the previous model in (10), we rewrite it in its discrete form as:

$$
e_{R R F}\left(A_{l}, A_{r}\right)=\sum_{i j \in \text { Image }}\left[P_{i j}-\left(g\left(\frac{X_{i}^{t} A_{l}-j}{s}\right)+\frac{1}{2}\right)\left(\frac{1}{2}-g\left(\frac{X_{i}^{t} A_{r}-j}{s}\right)\right)\right]^{2}(12)
$$

Again, we apply the Lagrange's formulation, which allows us to derive the associated iterative algorithm. As detailed in [33], to introduce constrains, the even function $g^{2}$ of the left and right residuals is rewritten as $g^{2}(t)=h\left(t^{2}\right)$, after expansion of the square in (12). Thus auxiliary variables $\omega_{i j}^{l}=\left(\frac{X_{i}^{t} A_{l}-j}{s}\right)$, $\omega_{i j}^{r}=\left(\frac{X_{i}^{t} A_{r}-j}{s}\right), \nu_{i j}^{l}=\left(\frac{X_{i}^{t} A_{l}-j}{s}\right)^{2}$ and $\nu_{i j}^{r}=\left(\frac{X_{i}^{t} A_{r}-j}{s}\right)^{2}$ are introduced. The associated Lagrange function is:

$$
\begin{aligned}
L_{R R F}= & \sum_{i j}\left[h\left(\nu_{i j}^{l}\right) h\left(\nu_{i j}^{r}\right)+\frac{1}{4}\left(h\left(\nu_{i j}^{l}\right)+h\left(\nu_{i j}^{r}\right)\right)+\left(2 P_{i j}-1\right) g\left(\omega_{i j}^{l}\right) g\left(\omega_{i j}^{r}\right)\right. \\
& \left.+\left(P_{i j}-1 / 4\right)\left[-g\left(\omega_{i j}^{l}\right)+g\left(\omega_{i j}^{r}\right)\right]-h\left(\nu_{i j}^{l}\right) g\left(\omega_{i j}^{r}\right)+h\left(\nu_{i j}^{r}\right) g\left(\omega_{i j}^{l}\right)\right] \\
& +\sum_{i j} \lambda_{i j}^{l}\left(\omega_{i j}^{l}-\frac{X_{i}^{t} A_{l}-j}{\sigma}\right)+\lambda_{i j}^{r}\left(\omega_{i j}^{r}-\frac{X_{i}^{t} A_{r}-j}{\sigma}\right) \\
& +\sum_{i j} \mu_{i j}^{l}\left(\nu_{i j}^{l}-\left(\frac{X_{i}^{t} A_{l}-j}{\sigma}\right)^{2}\right)+\mu_{i j}^{r}\left(\nu_{i j}^{r}-\left(\frac{j-X_{i}^{t} A_{r}-j}{\sigma}\right)^{2}\right)
\end{aligned}
$$

The derivatives of (13) w.r.t.: the auxiliary variables, the unknown variables $A_{l}$ and $A_{r}$, and the Khun-Tucker multipliers $\lambda_{i j}^{l}, \lambda_{i j}^{r}, \mu_{i j}^{l} \mu_{i j}^{r}$ are set to zero to obtain the algorithm. Like in the previous section, to improve convergence 
towards a local minima close to the global one, the optimization is iterated for several scales $s$.

The last images in first and second rows of Figure 7 show the resulting fit. We observed that fitting algorithms based on a region model are more robust to outliers compared to algorithm based on edges.

\section{Conclusion}

We presented the Lagrange's formulation of Half-Quadratic approach and showed how it can be used to derive the classical IRLS and MRLS algorithms for robustly estimating the parameters of a linear generative model, and to prove their convergence. Examples taken from road scene analysis illustrated the interest of these algorithms in difficult real-world applications such as lane marking detection and tracking or road sign detection and recognition. Other examples, namely simultaneous multiple lane fitting, road profile reconstruction and road region detection, demonstrated the flexibility of our framework which helps in the derivation of new algorithms. The obtained algorithms are quite fast: it takes around $0.2 \mathrm{~s}$ to fit simultaneously multiple lanes, around $0.5 \mathrm{~s}$ to reconstruction the road profile, and around $3 \mathrm{~s}$ to fit the road region, using a Core 2 Duo processor on images of size $640 \times 512$.

One may notice that the Lagrange's formulation of Half-Quadratic approach has interesting connections with the Expectation Maximization (EM) approach which will be interesting to investigate more in the future.

One difficulty remains to be solved, which is of practical importance in the context of road scene analysis since correlation matrices are useful for validating the fits. In all our derivation, we supposed independent noise. 
Our experience is that this assumption, which seems sufficient as long as only fitting is concerned, is not pertinent any more when estimating the correlation matrix of fits in practical applications. So the question is: how would it be possible to introduce correlated noise with heavy-tailed distribution within the Half-Quadratic approach?

\section{Acknowledgments}

Thanks to Pr Fabrice Heitz, Dr Rozenn Dahyot, Dr Sio-Song Ieng and Dr Erwan Bigorgne for their contributions.

\section{References}

[1] I. Mizera, C. Müller, Breakdown points and variation exponents of robust M-estimators in linear models, The Annals of Statistics 27 (4) (1999) 1164-1177.

[2] P. J. Huber, Robust Statistics, John Wiley and Sons, New York, New York, 1981.

[3] S. Geman, D. Geman, Stochastic relaxation, Gibbs distributions and the Bayesian restoration of images, IEEE Transactions on Pattern Analysis and Machine Intelligence 6 (6) (1984) 721-741.

[4] D. Geman, G. Reynolds, Constrained restoration and the recovery of discontinuities, IEEE Transactions on Pattern Analysis and Machine Intelligence 14 (3) (1992) 367-383. 
[5] D. Geman, C. Yang, Nonlinear image recovery with half-quadratic regularization and FFT's, IEEE Transactions on Image Processing 4 (7) (1995) 932-946.

[6] P. Charbonnier, L. Blanc-Féraud, G. Aubert, M. Barlaud, Two deterministic half quadratic regularization algorithms for computed imaging, in: Proceedings IEEE International Conference on Image Processing (ICIP'94), Vol. 2, Austin, TX, USA, 1994, pp. 168-172.

[7] P. Charbonnier, L. Blanc-Féraud, G. Aubert, M. Barlaud, Deterministic edge-preserving regularization in computed imaging, IEEE Transactions on Image Processing 6 (2) (1997) 298-311.

[8] M. J. Black, A. Rangarajan, On the unification of line processes, outlier rejection, and robust statistics with applications in early vision, International Journal of Computer Vision 19 (1) (1996) 57-92.

[9] K. Lange, D. R. Hunter, I. Yang, Optimization transfer using surrogate objective functions, Journal of Computational and Graphical Statistics 9 (1) (2000) 1-20.

[10] A. Yuille, A. Rangarajan, The concave-convex procedure, Neural Comput. 15 (4) (2003) 915-936.

[11] F. Champagnat, J. Idier, A connection between half-quadratic criteria and EM algorithms, IEEE Signal Processing Letters 11 (9) (2004) 709712 .

[12] J.-P. Tarel, S.-S. Ieng, P. Charbonnier, A constrained-optimization based half-quadratic algorithm for robustly fitting sets of linearly 
parametrized curves, Advances in Data Analysis and Classification 2 (3) (2008) 227-239.

[13] J.-P. Tarel, P. Charbonnier, S.-S. Ieng, A revisited half-quadratic approach for simultaneous robust fitting of multiple curves, in: H. A. J. Braz, A. Ranchordas, J. M. Pereira (Eds.), Computer vision and Computer Graphics, revised selected papers of visigrapp'07, CCIS 21, Springer-Verlag, Berlin Heidelberg, Germany, 2009, pp. 121-133.

[14] M. Minoux, Mathematical Programming: Theory and Algorithms, Chichester: John Wiley and Sons, 1986.

[15] D. G. Luenberger, Introduction to linear and nonlinear programming, Addison Wesley, 1973.

[16] J.-P. Tarel, S.-S. Ieng, P. Charbonnier, Using robust estimation algorithms for tracking explicit curves, in: European Conference on Computer Vision (ECCV'02), Vol. 1, Copenhagen, Danmark, 2002, pp. 492507.

[17] S.-S. Ieng, J.-P. Tarel, P. Charbonnier, Modeling non-Gaussian noise for robust image analysis, in: Proceedings of International Conference on Computer Vision Theory and Applications (VISAPP'07), Barcelona, Spain, 2007, pp. 175-182.

[18] S. Geman, D. McClure, Bayesian image analysis: an application to single photon emission tomography, Proc. Statistical Computational Section, Amer. Statistical Assoc. (1985) 12-18. 
[19] A. Blake, A. Zisserman, Visual Reconstruction, MIT Press, Cambridge, MA, 1987.

[20] S. S. Ieng, Méthodes robustes pour la détection et le suivi des marquages., Ph.D. thesis, Université Paris VI, France (2004).

[21] T. Veit, J.-P. Tarel, P. Nicolle, P. Charbonnier, Evaluation of road marking feature extraction, in: Proceedings of 11th IEEE Conference on Intelligent Transportation Systems (ITSC'08), Beijing, China, 2008, pp. $174-181$.

[22] F. Guichard, J.-P. Tarel, Curve extraction combining perceptual grouping and a kalman like fitting, in: IEEE International Conference on Computer Vision (ICCV'99), Kerkyra, Greece, 1999, pp. 1003-1008.

[23] J.-P. Tarel, P. Charbonnier, S.-S. Ieng, Simultaneous robust fitting of multiple curves, in: Proceedings of International Conference on Computer Vision Theory and Applications (VISAPP'07), Barcelona, Spain, 2007, pp. 175-182.

[24] M. Turk, A. Pentland, Eigenfaces for recognition, Journal of Cognitive Neuroscience 3 (1) (1991) 71-86.

[25] R. Dahyot, P. Charbonnier, F. Heitz, Robust Bayesian detection using appearance-based models, Pattern Analysis and Applications 7 (2004) $317-332$.

[26] T. Vik, F. Heitz, P. Charbonnier, Robust pose estimation and recognition using non-Gaussian modeling of appearance subspaces, IEEE 
Transactions On Pattern Analysis and Machine Intelligence 29 (5) (2007) 901-905.

[27] M. Tipping, C. Bishop, Probabilistic principal component analysis, Journal of the Royal Statistical Society, Series B 61 (3) (1999) 611-622.

[28] K. Fukunaga, L. Hostetler, The estimation of the gradient of a density function, with applications in pattern recognition, IEEE Transactions on Information Theory 21 (1975) 32-40.

[29] M. Fashing, C. Tomasi, Mean shift is a bound optimization, IEEE Transactions on Pattern Analysis and Machine Intelligence 27 (3) (2005) 471474.

[30] J.-P. Tarel, S.-S. Ieng, P. Charbonnier, Accurate and robust image alignment for road profile reconstruction, in: Proceedings of the IEEE International Conference on Image Processing (ICIP'07), Vol. V, San Antonio, Texas, USA, 2007, pp. 365-368.

[31] J.-P. Tarel, Global 3D planar reconstruction with uncalibrated cameras and rectified stereo geometry, International Jounal Machine Graphics and Vision 6 (4) (1997) 393-418.

[32] S. Granger, X. Pennec, Multi-scale EM-ICP: A fast and robust approach for surface registration, in: European Conference on Computer Vision (ECCV 2002), Vol. 2353 of LNCS, Springer, Copenhagen, Denmark, 2002, pp. 418-432.

[33] E. Bigorgne, J.-P. Tarel, Backward segmentation and region fitting for geometrical visibility range estimation, in: Proceedings of the Asian 
495 Conference on Computer Vision (ACCV'07), Vol. II, Tokyo, Japan, $496 \quad 2007$, pp. 817-826.

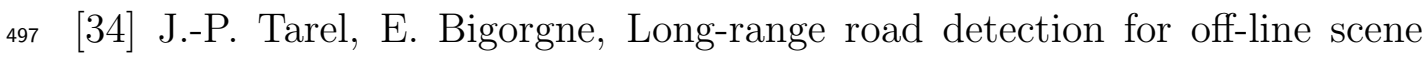
498 analysis, in: Proceedings of IEEE Intelligent Vehicle Symposium 499 (IV'2009), Xian, China, 2009, pp. 15-20. 